\title{
Inscription du patrimoine universitaire aux monuments historiques. Un outil pour l'avenir
}

\section{Baptiste Cottard}

\section{OpenEdition \\ Journals}

Édition électronique

URL : https://journals.openedition.org/ocim/4086

DOI : 10.4000/ocim.4086

ISSN : 2108-646X

Éditeur

OCIM

Édition imprimée

Date de publication : 1 septembre 2020

Pagination : 16-21

ISSN : 0994-1908

Référence électronique

Baptiste Cottard, «Inscription du patrimoine universitaire aux monuments historiques. Un outil pour l'avenir », La Lettre de l'OCIM [En ligne], 191 | 2020, mis en ligne le 01 septembre 2021, consulté le 12 décembre 2021. URL : http://journals.openedition.org/ocim/4086 ; DOl : https://doi.org/10.4000/ ocim. 4086

Ce document a été généré automatiquement le 12 décembre 2021.

Tous droits réservés 


\title{
Inscription du patrimoine universitaire aux monuments historiques. Un outil pour l'avenir
}

\author{
Baptiste Cottard
}

Phylogénie des Angiospermes illustrée présentée à l'Atheneum au printemps 2019 lors de l'exposition Anatomies. Papiers-mâchés - Pièces détachées.



(c) V. Arbelet/Mission Culture scientifique, université de Bourgogne 
1 En juin 2019, un ensemble exceptionnel provenant des fonds patrimoniaux de l'université de Bourgogne a été inscrit au titre des Monuments historiques (MH) par la Drac Bourgogne Franche-Comté. Première expérience à l'université de Bourgogne en ce qui concerne les objets mobiliers, ce n'est pas moins de 180 modèles botaniques et 8 modèles anatomiques qui jouissent désormais de cette protection.

2 Signés Auzoux et Brendel, deux fabricants des $\mathrm{XIX}^{\mathrm{e}}$ et $\mathrm{XX}^{\mathrm{e}}$ siècles, ces objets pédagogiques sont pour une grande majorité dans un très bon état de conservation. Issus des collections de biologie végétale et de médecine, ils ont été sauvegardés pour leur valeur esthétique, mais aussi didactique. S'ajoutent ainsi par le biais de ce nouveau statut, une double valeur historique et sémantique.

Si les modèles anatomiques sont aujourd'hui des vestiges de l'enseignement en médecine, au même titre que les cires, les modèles végétaux, eux, sont utilisés chaque année durant les cours de systématique.

\section{Les maisons Auzoux et Brendel}

Auzoux et Brendel sont deux des fabricants les plus connus de modèles pédagogiques en sciences naturelles durant le $\mathrm{XIX}^{\mathrm{e}}$ siècle et la première moitié du $X^{\mathrm{e}}$ siècle. Médecin de formation, Louis Thomas Jérôme Auzoux (1797-1880) propose, grâce à ses ateliers fondés en 1828, un catalogue exceptionnel de modèles allant de l'anatomie humaine et animale à la botanique. Robert Brendel (1821-1898) s'associe, lui, à un pharmacien et un botaniste pour la réalisation de plantes de grandes tailles. Le catalogue de la compagnie Brendel proposera à sa fermeture 220 modèles environ. Ces sculptures à la frontière des sciences et de l'art seront utilisées, pour leur grande précision, dans l'enseignement secondaire et supérieur pendant de nombreuses années et dans plusieurs pays d'Europe et du monde.

\section{Un outil de pérennisation et de légitimation}

Présents depuis plusieurs décennies dans les collections pédagogiques de l'université, ces modèles ont traversé - comme tant d'autres en France - la désaffection puis, sous l'impulsion de professeurs investis, un regain d'intérêt. Dans les années 1960 à Dijon, le professeur en médecine Pierre Barry constitue à ce titre une salle pédagogique dédiée à l'étude anatomique. Elle existe toujours aujourd'hui et présente à la fois les pièces Auzoux, des cires anatomiques Tramond, des pièces naturelles (os et dissections sèches) ainsi que des modèles en carton-pâte créés par Barry lui-même. De la même manière, les professeurs Michel Pauty, Michel Jannin et Nicole Autissier se sont intéressés quelques années plus tard aux collections de l'ancienne faculté des sciences, sauvant ainsi des dizaines d'instruments de la destruction. En biologie végétale, si aucune présentation particulière n'a été pensée pour les modèles Brendel et l'ensemble des planches botaniques, les professeurs Jean Vallade et Claude Humbert se penchent sérieusement sur ce fond dès les années 1980. La Mission Culture scientifique (service de l'université de Bourgogne dédié aux collections créé il y a plus de dix ans) poursuit cet engagement des personnels investis, le soutient sur l'ensemble du campus dijonnais et l'encadre. 
5 Souvent méconnues des professionnels de musées et des acteurs de l'enseignement supérieur, ces collections universitaires sont en constante construction. Disséminés dans les bureaux, les laboratoires ou encore les sous-sols, il est parfois difficile d'avoir une vision exhaustive des objets dont la sauvegarde présente un intérêt historique et scientifique. La prospection in situ devient alors l'un des moyens les plus sûrs pour enrichir les fonds. Commence ainsi un travail de sensibilisation auprès des équipes, puis d'expertise dans le domaine patrimonial. Des projets sont menés conjointement pour la protection et la valorisation de ces biens pour lesquels l'attachement des personnels de laboratoire relève parfois plus du sensible et de l'affect que de la raison. À ce titre, cette inscription aux $\mathrm{MH}$, comme les autres initiatives menées, représente à la fois un aboutissement pour cet engagement commun et un outil important de reconnaissance du service auprès de la communauté universitaire.

6 Au-delà d'une simple protection de l'intégrité physique, l'inscription est aussi « un gage pour l'avenir, afin d'éviter, un jour, leur dispersion " ${ }^{1}$ comme le précise Hélène Palouzié. Cela est particulièrement le cas pour les modèles botaniques Brendel reconnus et protégés comme "ensemble $"^{2}$. La série de plus de 180 modèles du fabricant allemand que la faculté possédait dans les années 1950 est aujourd'hui diminuée à 162 items. Environ 20 modèles ont été détruits ou perdus au cours des années. Ainsi, et par la même occasion, ce statut officiel prémunit la collection des inattentions du passé et permet de mettre en place des actions de conservation en adéquation avec les normes.

7 Par son aspect pérenne, la reconnaissance d'un statut particulier par le ministère de la Culture - représenté par la Drac - permet, de manière intrinsèque, de conscientiser l'importance de ces objets auprès de la communauté des enseignants et techniciens, en dépit de la précarité des postes au sein des équipes, problème que connaissent la majorité des institutions.

\section{Entre reconnaissance et appropriation}

8 La présentation des modèles auprès de la Drac durant plusieurs années en vue d'une inscription a permis d'élargir le champ des collections patrimoniales dont l'intérêt historique doit être sauvegardé, au même titre que les œuvres d'art. Ainsi les instruments scientifiques et les objets pédagogiques sont rares en commission et suscitent un intérêt particulier quand ils sont présentés. Si plusieurs modèles Auzoux, notamment animaux, ont déjà fait l'objet d'une inscription ou d'un classement en France, ce n'était pas le cas jusqu'à présent pour les modèles botaniques Brendel. Il s'agit donc d'un premier exemple en France de protection pour ces modèles. L'inscription au titre des Monuments historiques permet, à ce titre, d'offrir une reconnaissance officielle à cet héritage souvent mésestimé et mal documenté.

9 Avec ses 162 modèles botaniques fabriqués par Brendel et 18 par Auzoux, la collection de l'université de Bourgogne est l'une des plus importantes, avec celles des universités de Rennes, Lille et Strasbourg. Ces objets didactiques, au sens large - qu'ils apparaissent dans les collections de médecine, d'école vétérinaire ou de botanique - connaissent depuis plusieurs années un regain d'intérêt avec de multiples expositions, journées d'études ou encore projets de restauration médiatisés. 
L'objectif, en présentant une cinquantaine de modèles dans la salle "Brendel/Auzoux : artisans de la nature ", était de privilégier la dimension esthétique et sensible à l'aspect scientifique (Anatomies, 2019).



(c) V. Arbelet/Mission Culture scientifique, université de Bourgogne

Les objets pédagogiques s'exposent de plus en plus. Pour Anatomies (2019), les modèles de la collection sont remis dans le contexte scientifique de leur utilisation pour l'enseignement de la biologie végétale.

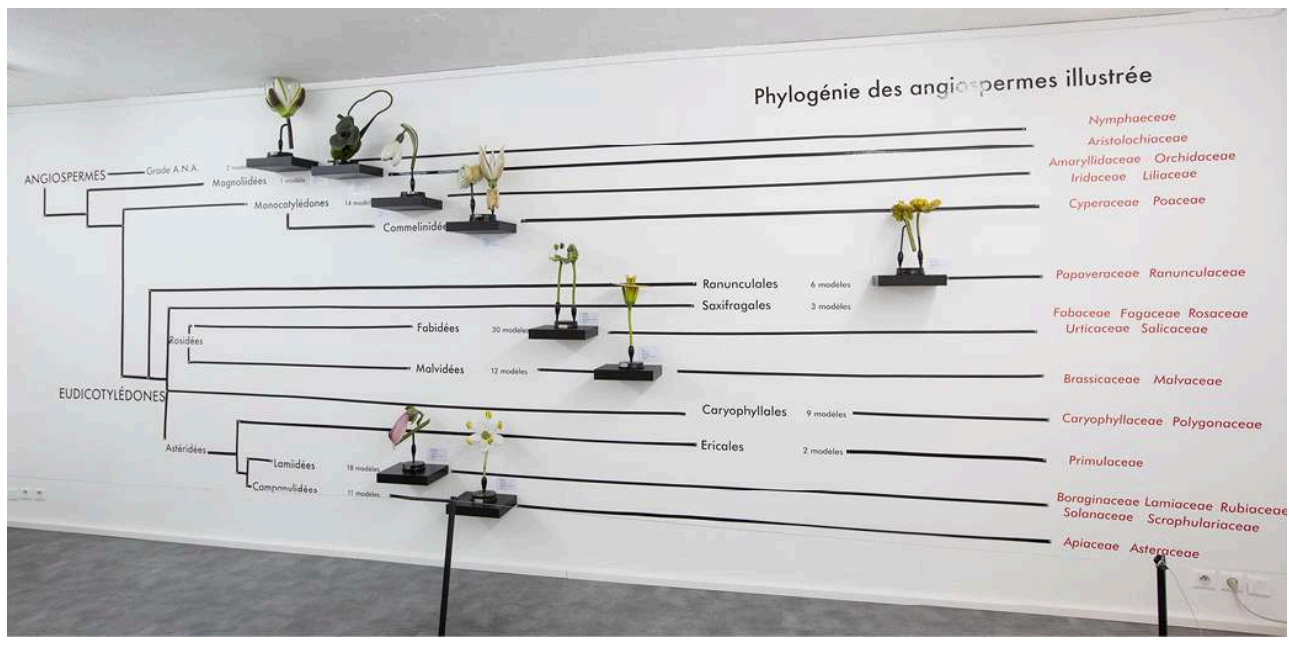

(C) V. Arbelet/Mission Culture scientifique, université de Bourgogne

10 Une rencontre professionnelle ${ }^{3}$ autour des modèles d'enseignement en botanique a notamment été l'occasion de présenter la collection de l'université de Bourgogne à travers ses modèles, herbiers et planches anatomiques. L'intervention, basée sur un travail mené conjointement par le service et une enseignante en biologie végétale, a permis de souligner, au-delà du simple intérêt de conservation physique des modèles, la pertinence de ces supports dans l'enseignement. En effet, au même titre que l'université de Lille, les modèles de Dijon sont encore utilisés pour l'étude de l'anatomie des plantes et des fleurs. Ils permettent, en complément des observations au microscope, de conceptualiser en trois dimensions l'organisation des organes et leur fonctionnement. 
11 Le parti pris, dans ce cas précis, est de faire vivre ces objets pour leur fonction d'origine et non pas de les faire sortir du circuit de l'apprentissage pour les présenter sous vitrine. Objets pédagogiques avant tout, ces modèles inscrits continueront à être utilisés par le corps enseignant. La condition de survie du patrimoine universitaire n'étant pas seulement d'avoir des professionnels dédiés à sa conservation, mais aussi et surtout de susciter ce phénomène d'appropriation par la communauté universitaire. Il est important, pour les objets inventoriés comme pour ceux qui ne le sont pas encore, d'impliquer à la fois les enseignants, les techniciens de laboratoire, les ingénieurs de recherche et les étudiants.

La découverte et la sensibilisation sont au cœur des actions de valorisation proposées par la Mission Culture scientifique. Des expositions régulières organisées au sein du centre culturel du campus permettent de valoriser des objets de collections sur des thématiques différentes, privilégiant un lien fort entre art et sciences. Ainsi, au printemps 2019, une exposition présentait pour la première fois environ 80 modèles botaniques et anatomiques Brendel et Auzoux pour leur qualité esthétique et leur place dans l'histoire de l'enseignement ${ }^{4}$.

13 L'évènement, centré sur le matériau papier-mâché, donnait à voir l'histoire des deux fabricants, leur formation et leur méthode de travail. L'habileté technique et le temps de travail dédié à la création de ces modèles, des données qui peuvent paraître anecdotiques au regard du pragmatisme de l'objet scientifique, ont révélé une dimension sensible à laquelle les spectateurs se sont montrés réceptifs. Cette dimension, mêlée à l'esthétique des formes et des couleurs, est l'un des facteurs principaux du sentiment d'appropriation que le personnel de l'université a pu ressentir et exprimer lors des visites guidées. Ainsi, l'inscription des modèles aux MH annoncée durant la période de l'exposition, ne paraissait que justifiée auprès du public.

L'Homme complet clastique du Dr Auzoux, signé et daté de 1884 est présenté non restauré pour aborder l'importance de la conservation des collections universitaires (Anatomies, 2019).



(C) V. Arbelet/Mission Culture scientifique, université de Bourgogne 


\section{Des modèles au cours de systématique}

L'espace scénographique de l'exposition Anatomies incluait une phylogénie illustrée des Angiospermes, classification des plantes à fleurs par grandes familles. Basé sur le travail de Beryl Laitung, maître de conférences en biologie et écologie végétale, l'objectif de cette présentation était de souligner la pertinence de l'utilisation des collections pour les cours de systématique aujourd'hui. Malgré leur siècle et demi d'ancienneté et l'évolution de cette classification, les pièces conservées à Dijon représentent à elles seules 67 des 150 familles de plantes à fleurs répertoriées en France.

Sur les 25 principales familles de la flore française (inscrites en rouge), 9 d'entre elles étaient représentées ici par un modèle des collections. Pour chacune d'entre elles, le nombre de modèles présents dans l'ensemble était lui aussi mentionné sur le mur pour en souligner la richesse.

Affiche de l'exposition Anatomies. Papiers-mâchés - Pièces détachées présentée à l'Atheneum (2019).

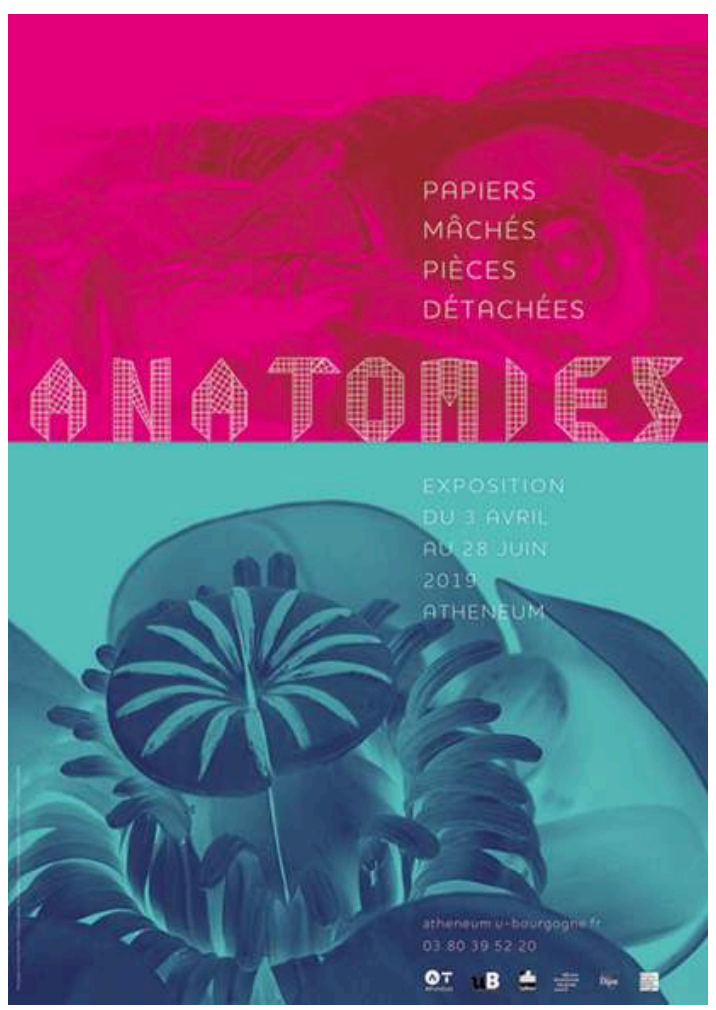

(c) Mission Culture scientifique, université de Bourgogne

14 À l'image des musées communiquant sur leurs pratiques internes, une partie de cette exposition était consacrée au travail de protection et de valorisation des collections sur l'université. Autour d'un modèle anatomique Auzoux présenté volontairement dans son état catastrophique de conservation, l'objectif était encore une fois de sensibiliser et de souligner l'importance du travail du service.

Cette première expérience au sein de l'université de Bourgogne n'est pas qu'une simple assurance de la survie de ces objets. Bien plus encore, c'est l'illustration même de 
l'expertise patrimoniale, voie sur laquelle s'engagent progressivement les services dédiés aux collections dans les universités. Chantiers de collections, conventions de prêts et constats d'états, autant d'outils du monde muséal qui s'adaptent aux conditions qu'offrent ces collections particulières. Malgré cela, il ne s'agit pas de rendre inaccessibles des objets dont l'utilité est primordiale, mais de prendre conscience des spécificités de ces ensembles et d'y appliquer des règles de conduite.

\section{NOTES}

1. Palouzié H. La protection Monument historique : connaissance et reconnaissance des collections de l'université de Montpellier, In situ - Revue des patrimoines, n¹7, 2011.

2. "Un ensemble ou une collection d'objets mobiliers dont la conservation dans son intégrité et sa cohérence présente un intérêt public au point de vue de l'histoire, [...], de la science ou de la technique peut être classé au titre des monuments historiques comme ensemble historique mobilier par décision de l'autorité administrative ", Article L 622-2 de l'ordonnance n²017-651 du 27 avril 2017 relative aux immeubles et objets mobiliers classés ou inscrits au titre des monuments historiques.

3. Journées d'études La botanique et ses objets d'enseignement, organisées par le Musée national de l'Éducation (Munaé) les 7 et 8 février 2019 à Rouen.

4. Exposition Anatomies. Papiers-mâchés - Pièces détachées présentée à l'Atheneum, centre culturel de l'université de Bourgogne, du 3 avril au 28 juin 2019.

\section{RÉSUMÉS}

La Mission Culture scientifique de l'université de Bourgogne a inscrit un ensemble patrimonial de matériel pédagogique au titre des Monuments historiques. Ainsi, elle souhaite montrer l'importance de cet héritage scientifique et s'appuyer sur ce statut pour valoriser les collections.

\section{INDEX}

Mots-clés : patrimoine et/ou collection universitaires ; organisation de l'enseignement supérieur ; musée universitaire

\section{AUTEUR}

\section{BAPTISTE COTTARD}

Chargé des collections muséales à l'université de Bourgogne et diplômé de muséologie à l'École du Louvre. 
baptiste.cottard@u-bourgogne.fr 